\title{
Accuracy Evaluation of Phase-field Models for Grain Growth Simulation with Anisotropic Grain Boundary Properties
}

\author{
Eisuke MIYOSHI, ${ }^{1)}$ Tomohiro TAKAKI, ${ }^{2) *}$ Munekazu OHNO ${ }^{3)}$ and Yasushi SHIBUTA ${ }^{4)}$ \\ 1) Graduate School of Science and Technology, Kyoto Institute of Technology, Matsugasaki, Sakyo-ku, Kyoto, $606-8585$ Japan. \\ 2) Faculty of Mechanical Engineering, Kyoto Institute of Technology, Matsugasaki, Sakyo-ku, Kyoto, 606-8585 Japan. \\ 3) Division of Materials Science and Engineering, Faculty of Engineering, Hokkaido University, Kita 13 Nishi 8, Kita-ku, \\ Sapporo, Hokkaido, 060-8628 Japan. \\ 4) Department of Materials Engineering, The University of Tokyo, 7-3-1 Hongo, Bunkyo-ku, Tokyo, $113-8656$ Japan.
}

(Received on May 17, 2019; accepted on July 10, 2019; J-STAGE Advance published date: September 7, 2019)

\begin{abstract}
The phase-field method has been widely employed recently for simulating grain growth. Phase-field grain growth models are classified into two types according to their conservation constraints for phase-field variables: the multi-phase-field model and the continuum-field model. In addition, within the multi-phase-field model framework, three models with different formulations exist. These models are reported to accurately simulate grain growth under conditions of isotropic or weakly anisotropic grain boundary energy and mobility. However, for cases of strongly anisotropic grain boundary properties, the accuracy of these models has not yet been examined in detail. In this study, using the continuum-field model and three different multi-phase-field models, systematic grain growth simulations with anisotropic grain boundary energies and mobilities are performed. Through the detailed investigation of the accuracy of the simulated results, the suitability of each model for anisotropic grain growth simulations is revealed. Furthermore, based on the higher-order terms, accuracy improvement of the phase-field models is attempted.
\end{abstract}

KEY WORDS: phase-field model; grain growth; microstructure; grain boundary energy; grain boundary mobility.

\section{Introduction}

The process of competitive grain growth under curvature-driven boundary migration is one of the most important microstructural evolution phenomena in the manufacturing of polycrystalline materials because it determines the final microstructures and resultant physical properties of materials. ${ }^{1-3)}$ However, the experimental study of grain growth is severely limited by the high temperatures at which it occurs and the optical opacity of polycrystalline samples. Therefore, to systematically examine grain growth phenomena, numerical approaches have been developed using continuum-based grain growth models, ${ }^{4)}$ including the Monte-Carlo, ${ }^{5-8)}$ cellular automaton, ${ }^{8-11)}$ vertex or front-tracking, ${ }^{12-17)}$ surface evolver, ${ }^{18-20}$ ) and phase-field ${ }^{7,21-26)}$ models. In particular, the phase-field method has become common because it can accurately address curvature-driven grain boundary migration without explicit boundary tracking. With the benefit of rapid developments in high-performance computing, recent phase-field simulations have enabled significant contributions toward elucidating various metallurgical processes, including grain

\footnotetext{
* Corresponding author: E-mail: takaki@kit.ac.jp

DOI: https://doi.org/10.2355/isijinternational.ISIJINT-2019-305
}

growth. $^{27-34)}$

Most phase-field studies on grain growth have focused on ideal grain growth under the conditions of isotropic grain boundary energy and mobility. However, in actual materials, these grain boundary properties are usually strongly anisotropic, with largely different values for each grain boundary. 1,35-37) Theoretical, experimental, and numerical studies have suggested that grain boundary property anisotropy is a dominant factor of important metallurgical phenomena related to grain growth, e.g., nucleation of recrystallized grains, ${ }^{38-40)}$ texture development, ${ }^{41-43)}$ preferential selection of specific grain boundaries, ${ }^{44-46)}$ and deviation of grain growth kinetics from the ideal parabolic law. ${ }^{47,48)}$ Therefore, to achieve more realistic prediction of microstructural evolutions, a phase-field model must be developed that can accurately simulate anisotropic boundary properties.

The most frequently used phase-field grain growth models are so-called multi-order-parameter models, which represent polycrystalline systems by assigning phase-field variables to each grain. These models can be classified into two types. One is the continuum-field (CF) model, in which the sum of the phase fields at each spatial point is non-conserved. This model originates from the works of Chen et al., ${ }^{49-51)}$ and was improved by Kazaryan et al. ${ }^{52)}$ and Moelans et al. ${ }^{53,54)}$ 
to quantitatively relate the model parameters to material properties. The other is the multi-phase-field (MPF) model with the constraint of phase-field conservation, which was first reported by Steinbach et al ${ }^{55)}$ and then reformulated by Garcke et al. $^{56)}$ and Steinbach and Pezzolla ${ }^{57)}$ to accommodate the treatment of multiple-grain interactions at multi-junctions. In addition, a simplified version of the MPF model ${ }^{58)}$ referred to as the anti-symmetric MPF (ASMPF) model, has been widely employed for its computational convenience. Furthermore, within the framework of the MPF model, a recent work by Tóth et al. ${ }^{59)}$ proposed a generalized MPF (XMPF) model based on a unique formulation. All of these phase-field models are reportedly capable of accurate simulation of grain growth under isotropic or weakly anisotropic grain boundary properties (e.g., the ratio of the maximum and minimum boundary energies is less than two $\left.{ }^{60-63)}\right)$. However, for cases with strong anisotropy in grain boundary properties, the accuracy of the models has not yet been tested in detail.

Based on the above background, this study performs a detailed evaluation of the accuracies of the four different phase-field models (CF, MPF, ASMPF, and XMPF), elucidating the applicability of each model to the simulation of grain growth with strongly anisotropic grain boundary energy and mobility. The rest of this paper is outlined as follows: first, Section 2 briefly describes the formulations of the phase-field grain growth models. Next, Section 3 examines the effects of large differences in boundary properties on the accuracy of the models by performing a series of grain growth simulations for an idealized tri-crystal system. Finally, in the same section, we discuss a way to improve the accuracy based on the higher-order modification. ${ }^{63-68)}$

\section{Phase-field Grain Growth Models}

All of the CF, MPF, ASMPF, and XMPF models represent polycrystalline systems consisting of $N$ grains through $N$ phase-field variables $\phi_{i}(i=1,2, \ldots, N)$, which take a value of 1 in the $i$ th grain, 0 in the other grains, and $0<\phi_{i}<1$ at the grain boundaries. The free energy functional of the system is defined as:

$$
F=\int_{V}\left[f_{\text {barr }}(\phi)+f_{\text {grad }}(\phi, \nabla \phi)\right] \mathrm{d} V,
$$

where $f_{\text {barr }}$ and $f_{\text {grad }}$ indicate the barrier term and gradient term, respectively. Based on this free energy functional, in all models, the migration of grain boundaries is reproduced by calculating the time evolution of $\phi_{i}$ for each spatial point under the constraints of free energy minimization. However, the specific forms of $f_{\text {barr }}$ and $f_{\text {grad }}$ and the derivation of the time-evolution equation differ significantly for each model, as described below.

\subsection{CF Model}

In the $\mathrm{CF}$ model, ${ }^{53)}$ the barrier term $f_{\text {barr }}$ and gradient term $f_{\text {grad }}$ are given as:

$$
\begin{aligned}
& f_{\text {barr }}=m\left\{\sum_{i=1}^{N}\left(\frac{\phi_{i}^{4}}{4}-\frac{\phi_{i}^{2}}{2}\right)+\sum_{i=1}^{N} \sum_{j>i}^{N} w_{i j} \phi_{i}^{2} \phi_{j}^{2}+\frac{1}{4}\right\}, \\
& f_{\text {grad }}=\sum_{i=1}^{N} \frac{\kappa}{2}\left(\nabla \phi_{i}\right)^{2}, \kappa=\sum_{i=1}^{N} \sum_{j>i}^{N} \varepsilon_{i j} \phi_{i}^{2} \phi_{j}^{2} / \sum_{i=1}^{N} \sum_{j>i}^{N} \phi_{i}^{2} \phi_{j}^{2},
\end{aligned}
$$

where $m$ is a parameter and $w_{i j}$ and $\varepsilon_{i j}$ denote the barrier height and gradient coefficient of the grain boundary between the $i$ th and $j$ th grains, respectively. Here, $f_{\text {barr }}$ is formulated based on the so-called "multi-well" potential. The time-evolution equation of $\phi_{i}$ is derived as follows without the constraint of phase-field conservation:

$$
\begin{aligned}
& \frac{\partial \phi_{i}}{\partial t}=-L \frac{\delta F}{\delta \phi_{i}}, \\
& \frac{\delta F}{\delta \phi_{i}}=m\left(\phi_{i}^{3}-\phi_{i}+2 \phi_{i} \sum_{j \neq i}^{N} w_{i j} \phi_{j}^{2}\right)-\kappa \nabla^{2} \phi_{i}, \\
& L=\sum_{i=1}^{N} \sum_{j>i}^{N} L_{i j} \phi_{i}^{2} \phi_{j}^{2} / \sum_{i=1}^{N} \sum_{j>i}^{N} \phi_{i}^{2} \phi_{j}^{2},
\end{aligned}
$$

where $L_{i j}$ is the phase-field mobility of the $i-j$ grain boundary. The model parameters $m, w_{i j}, \varepsilon_{i j}$, and $L_{i j}$ can be related to the width $\left(\delta_{\mathrm{MW}}\right.$, where the subscript MW indicates the multi-well potential), energy $\left(\sigma_{i j}\right)$, and mobility $\left(M_{i j}\right)$ of the grain boundary by using the numerical algorithm proposed by Moelans et al. ${ }^{53)}$ While the original algorithm of Moelans et al. uses the fixed grain boundary width $\delta_{\mathrm{MW}}$ for all boundaries, Tang et al. ${ }^{69)}$ proposed a modification that varies $\delta_{\mathrm{MW}}$ for each boundary depending on its energy $\sigma_{i j}$. From our preliminary tests, the modified algorithm yielded better computational accuracy for the cases where two high-energy boundaries and one low-energy boundary are connected at the triple junction (corresponding to the conditions of the energy ratio $\sigma_{\mathrm{A}} / \sigma_{\mathrm{B}}<1$ in Fig. 1; see Section 3 for details). Therefore, for such cases, we employ the algorithm of Tang et al.

\subsection{MPF and ASMPF Models}

The MPF model ${ }^{57)}$ has the following forms of $f_{\text {barr }}$ and $f_{\text {grad: }}$

$$
f_{\text {barr }}=\sum_{i=1}^{N} \sum_{j>i}^{N} w_{i j} \phi_{i} \phi_{j}, f_{\text {grad }}=-\sum_{i=1}^{N} \sum_{j>i}^{N} \frac{\varepsilon_{i j}^{2}}{2} \nabla \phi_{i} \cdot \nabla \phi_{j} \ldots \ldots . .
$$
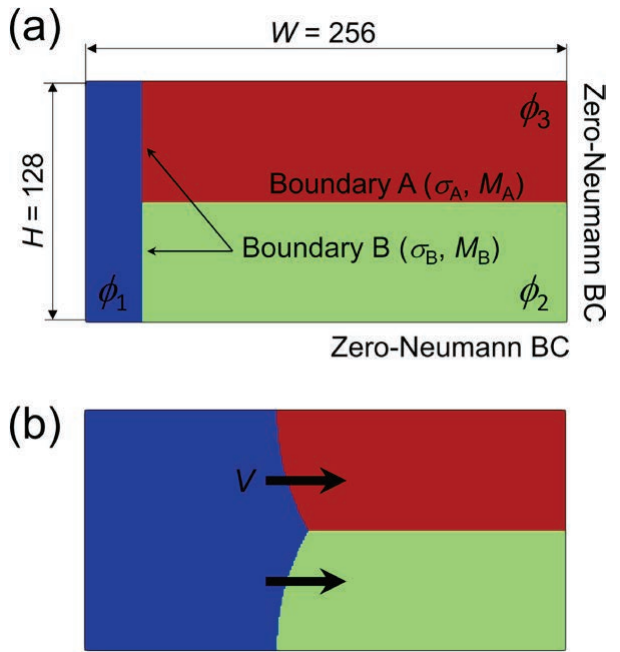

Fig. 1. Tri-crystal system for evaluating the accuracy of the phase-field grain growth models: (a) initial state and (b) steady state. The system consists of three grains represented by the phase-field variables $\phi_{1}, \phi_{2}$, and $\phi_{3}$. In each panel, grains are distinguished by different colors. (Online version in color.) 
The barrier term $f_{\text {barr }}$ in Eq. (4) is generally referred to as the "double-obstacle" potential. In this model, the sum of the phase-field variables at any spatial point in the system must be conserved as $\sum_{i=1}^{N} \phi_{i}=1$. Under this constraint, the time-evolution equation is derived as:

$$
\frac{\partial \phi_{i}}{\partial t}=-\frac{2}{n} \sum_{j=1}^{n} L_{i j}\left(\frac{\delta F}{\delta \phi_{i}}-\frac{\delta F}{\delta \phi_{j}}\right), \quad \frac{\delta F}{\delta \phi_{i}}=\sum_{k=1}^{n}\left(w_{i k} \phi_{k}+\frac{1}{2} \varepsilon_{i k}^{2} \nabla^{2} \phi_{k}\right) \text {, }
$$

where $n$ is the number of nonzero phase fields at a given point. In this study, the $n$ values for each point are evaluated using the iterative algorithm of Takaki et al. ${ }^{70)}$ Our preliminary tests showed that this algorithm significantly improves the numerical accuracy and stability of MPF simulations. The parameters $w_{i j}, \varepsilon_{i j}$, and $L_{i j}$ can be directly related to the grain boundary properties through the following analytical equations:

$$
w_{i j}=4 \frac{\sigma_{i j}}{\delta_{\mathrm{DO}}}, \varepsilon_{i j}^{2}=\frac{8}{\pi^{2}} \delta_{\mathrm{DO}} \sigma_{i j}, L_{i j}=\frac{\pi^{2}}{8} \frac{M_{i j}}{\delta_{\mathrm{DO}}},
$$

where $\delta_{\mathrm{DO}}$ indicates the grain boundary width for the double-obstacle potential-based models.

The time-evolution equation for the ASMPF model ${ }^{58)}$ is obtained by applying the "antisymmetric approximation" "58,71) to the MPF Eq. (5), in which all terms of $k \neq i, j$ indices are neglected:

$$
\frac{\partial \phi_{i}}{\partial t}=\sum_{j=1}^{n} L_{i j}\left\{w_{i j}\left(\phi_{i}-\phi_{j}\right)+\varepsilon_{i j}^{2}\left(\phi_{j} \nabla^{2} \phi_{i}-\phi_{i} \nabla^{2} \phi_{j}\right)\right\}
$$

\subsection{XMPF Model}

In the XMPF model, ${ }^{59)} f_{\text {barr }}$ and $f_{\text {grad }}$ are defined as:

$$
\begin{aligned}
f_{\text {barr }} & =\omega\left\{\sum_{i=1}^{N}\left(\frac{\phi_{i}^{4}}{4}-\frac{\phi_{i}^{3}}{3}\right)+\frac{1}{2} \sum_{i=1}^{N} \sum_{j>i}^{N} \phi_{i}^{2} \phi_{j}^{2}+\frac{1}{12}\right\}, \\
\omega & =\sum_{i=1}^{N} \sum_{j>i}^{N} w_{i j} \phi_{i}^{2} \phi_{j}^{2} / \sum_{i=1}^{N} \sum_{j>i}^{N} \phi_{i}^{2} \phi_{j}^{2}, \\
f_{\text {grad }} & =\sum_{i=1}^{N} \frac{\kappa}{2}\left(\nabla \phi_{i}\right)^{2}, \kappa=\sum_{i=1}^{N} \sum_{j>i}^{N} \varepsilon_{i j}{ }^{2} \phi_{i}^{2} \phi_{j}^{2} / \sum_{i=1}^{N} \sum_{j>i}^{N} \phi_{i}^{2} \phi_{j}^{2} .
\end{aligned}
$$

Here, the multi-well-type potential is used for $f_{\text {barr }}$. With the constraint of phase-field conservation and the Bollada-Jimack-Mullis mobility matrix, ${ }^{65)} \Gamma$, the time-evolution equation of $\phi_{i}$ is derived as:

$$
\begin{aligned}
\frac{\partial \phi_{i}}{\partial t}= & -\sum_{j=1}^{N} \Gamma\left(\frac{\delta F}{\delta \phi_{i}}-\frac{\delta F}{\delta \phi_{j}}\right), \\
\frac{\delta F}{\delta \phi_{i}}= & \omega \frac{\partial f_{\text {barr }}}{\partial \phi_{i}}+\frac{\partial \omega}{\partial \phi_{i}} f_{\text {barr }}-\kappa \nabla^{2} \phi_{i} \\
& +\sum_{k=1}^{N}\left[\frac{1}{2} \frac{\partial \kappa}{\partial \phi_{i}} \nabla \phi_{k}-\frac{\partial \kappa}{\partial \phi_{k}} \nabla \phi_{i}\right] \cdot \nabla \phi_{k}, \\
\Gamma\left(\phi_{i}, \phi_{j}\right)= & L_{i j}\left|\frac{\phi_{i}}{1-\phi_{i}}\right|\left|\frac{\phi_{j}}{1-\phi_{j}}\right| .
\end{aligned}
$$

The analytical relations between the model parameters and grain boundary properties are given as:

$$
w_{i j}=24 \frac{\sigma_{i j}}{\delta_{\mathrm{MW}}}, \varepsilon_{i j}^{2}=\frac{3}{2} \delta_{\mathrm{MW}} \sigma_{i j}, L_{i j}=\frac{1}{3} \frac{M_{i j}}{\delta_{\mathrm{MW}}} \ldots
$$

\subsection{Grain Boundary Width in Each Model}

Although the above phase-field models all treat the grain boundaries as diffuse interfaces, the phase-field profiles at the interfaces differ for each model depending on the form of the barrier term $f_{\text {barr }}$. In the MPF and ASMPF models, which use the double-obstacle potential for $f_{\text {barr, }}$ the one-dimensional equilibrium profile of a phase field, $\phi_{i}(x)$, is given by a sine curve:

$$
\phi_{i}(x)=\frac{1}{2}\left[1-\sin \left(\pi \frac{x}{\delta_{\mathrm{DO}}}\right)\right] .
$$

In this case, the grain boundary width can be clearly defined as $\delta_{\mathrm{DO}}$, within which $\phi_{i}(x)$ has a value of $0<\phi_{i}<$ 1. On the other hand, for the multi-well-potential-based CF and XMPF models, the phase-field profiles $\phi_{i}(x)$ have infinite tails; the boundary width, $\delta_{\mathrm{MW}}$, for these models is typically defined by the gradient of $\phi_{i}$ at the center $(x=0)$ of the boundary:

$$
\delta_{\mathrm{MW}}=\frac{1}{\left|\left(\partial \phi_{i} / \partial x\right)_{x=0}\right|} .
$$

Using this definition of $\delta_{\mathrm{MW}}$, the one-dimensional equilibrium profile of $\phi_{i}$ can be expressed by a hyperbolic tangent function:

$$
\phi_{i}(x)=\frac{1}{2}\left[1-\tanh \left(2 \frac{x}{\delta_{\mathrm{MW}}}\right)\right] .
$$

Note that when the grain boundary properties are anisotropic, the curves of the phase-field profiles of the CF model deviate from the hyperbolic tangent curve, becoming more asymmetric in shape. ${ }^{53)}$

\section{Accuracy Evaluation of the Phase-field Models}

\subsection{Computational System and Parameters}

By performing grain growth simulations with anisotropic grain boundary energies and mobilities, we evaluate the accuracy of the above-described phase-field models. Here, time, spatial length, boundary energy, and mobility in the simulations are all given in dimensionless units scaled by typical orders of $1 \mathrm{~s}, 10^{-6} \mathrm{~m}, 1 \mathrm{~J} \cdot \mathrm{m}^{-2}$, and $10^{-12} \mathrm{~m}^{4} \cdot \mathrm{J}^{-1} \cdot \mathrm{s}^{-1}$, respectively. As the simulation model, an idealized tri-crystal system depicted in Fig. 1(a) is employed; the steady-state grain boundary velocity, $V$, shown in Fig. 1(b), is compared to the theoretical value. The computational system consists of three grains represented by different phase fields $\phi_{1}, \phi_{2}$, and $\phi_{3}$ that are under zero-Neumann boundary conditions in all directions. The domain with a height $H=128$ and width $W=256$ is divided into square regular grids of size $\Delta x$. The theoretical value of $V, V_{\mathrm{th}}$, is given as: ${ }^{40,60,72)}$

$$
V_{\mathrm{th}}=M_{\mathrm{B}} \frac{\sigma_{\mathrm{A}}}{H},
$$

where $\sigma_{\mathrm{A}}$ is the energy of grain boundary $\mathrm{A}$ and $M_{\mathrm{B}}$ is the mobility of boundary B. In the simulations, the energy of grain boundary $\mathrm{B}, \sigma_{\mathrm{B}}$, and the mobility of boundary $\mathrm{A}, M_{\mathrm{A}}$, are fixed to 1 , while $\sigma_{\mathrm{A}}$ and $M_{\mathrm{B}}$ are varied. In the conditions 
of $\sigma_{\mathrm{A}} / \sigma_{\mathrm{B}} \geq 2$, steady-state grain boundary migration cannot occur because grain boundary A splits into two boundaries under the occurrence of solid-state wetting. ${ }^{73)}$ Therefore, we limit the $\sigma_{\mathrm{A}}$ value such that $\sigma_{\mathrm{A}} / \sigma_{\mathrm{B}} \leq 1.9$. The time-evolution equations are numerically solved using the first-order forward difference scheme and the second-order central difference scheme for time and space, respectively. The time increment $\Delta t$ is taken as large as possible so as to satisfy the stability condition of the explicit scheme.

In general, the computational accuracy of a phase-field model is affected by the grid resolution and the grain boundary width. Herein, to determine the appropriate settings to evaluate the accuracies of the phase-field models, we first performed grain growth simulations while varying the resolution, $H / \Delta x$, and grain boundary width, through which the variations in the relative error $\left(V-V_{\text {th }}\right) / V_{\text {th }}$ were determined for each model. Here, the grain boundary properties were set to be isotropic $\left(\sigma_{\mathrm{A}}=\sigma_{\mathrm{B}}\right.$ and $\left.M_{\mathrm{A}}=M_{\mathrm{B}}\right)$. Note that, as described in Section 2.4, the definition of the grain boundary width is different for the MPF/ASMPF models $\left(\delta_{\mathrm{DO}}\right)$ and the $\mathrm{CF} / \mathrm{XMPF}$ models $\left(\delta_{\mathrm{MW}}\right)$. To directly compare the results for each model, we here employed the following quantity, $\delta$, as a unified measure of the boundary width:

$$
\delta=\delta_{\mathrm{DO}}=\frac{\pi}{2} \delta_{\mathrm{MW}} .
$$

When the same value is set to $\delta$, the gradients of phase fields at the centers of the grain boundaries are identical in each model. Here, we want to briefly note the actual grain boundary width ${ }^{74)}$ of each model, within which two or more phase fields take values larger than a small threshold value $\lambda$. It can be analytically shown from Eqs. (11) and (13) that, at the same value of $\delta$, the actual boundary width for the $\mathrm{CF} / \mathrm{XMPF}$ models is always larger than that for the MPF/ ASMPF models $;{ }^{74)}$ for instance, the ratio of the former and the latter is $1.185,1.677$, and 2.291 for the threshold values $\lambda=0.1,0.01$, and 0.001 , respectively. When we use the efficient numerical algorithm (so-called "active parameter tracking,"22,70,75,76) ) storing and solving only phase-field variables larger than $\lambda$, such difference in actual boundary width could lead to a significant difference in the computational costs of the models, especially in simulations for threedimensional complicated microstructures. The efficiencies of the models for such applications will be addressed in detail elsewhere.

The simulation results for different $H / \Delta x$ and $\delta$ values are shown in Fig. 2, from which we can see that $\delta=4 \Delta x$ yields more than $10 \%$ error for any $H / \Delta x$ and any phasefield model. In the cases of $\delta=8 \Delta x$ and $10 \Delta x$ for $H / \Delta x=$ 128 , the XMPF model shows good accuracy with less than $1 \%$ error. However, for the CF, MPF, and ASMPF models, these $\delta$ values yield errors of $3-5 \%$ even for $H / \Delta x=128$ and, therefore, are considered to be too large compared to the grid resolution of the system. In this case, although the errors decrease with the increase in $H / \Delta x$, it is expected that a very high resolution is necessary to decrease the errors to approximately zero. In contrast, the results obtained for $\delta=6 \Delta x$ are visibly more accurate, and the errors of the phase-field models all become less than $\sim 1 \%$ at $H / \Delta x=$ 128. Therefore, to optimize the computational accuracy and cost, we used $\delta=6 \Delta x$ and $H / \Delta x=128$ for the following simulations.

\subsection{Effect of Anisotropic Grain Boundary Energy}

To examine the effects of anisotropic grain boundary energy on the accuracies of the phase-field models, simulations were performed under the above-described conditions while varying the energy ratio $\sigma_{\mathrm{A}} / \sigma_{\mathrm{B}}$, with $M_{\mathrm{B}} / M_{\mathrm{A}}$ fixed to 1 . Figure 3 shows the relative errors $\left(V-V_{\text {th }}\right) / V_{\text {th }}$ of different models as functions of $\sigma_{\mathrm{A}} / \sigma_{\mathrm{B}}$. As shown in the figure, for the range of $\sigma_{\mathrm{A}} / \sigma_{\mathrm{B}} \geq 0.2$, although the error of the MPF model almost monotonically increases on the positive side as $\sigma_{\mathrm{A}} / \sigma_{\mathrm{B}}$ deviates from 1 , the error remains less than $\sim 10 \%$.

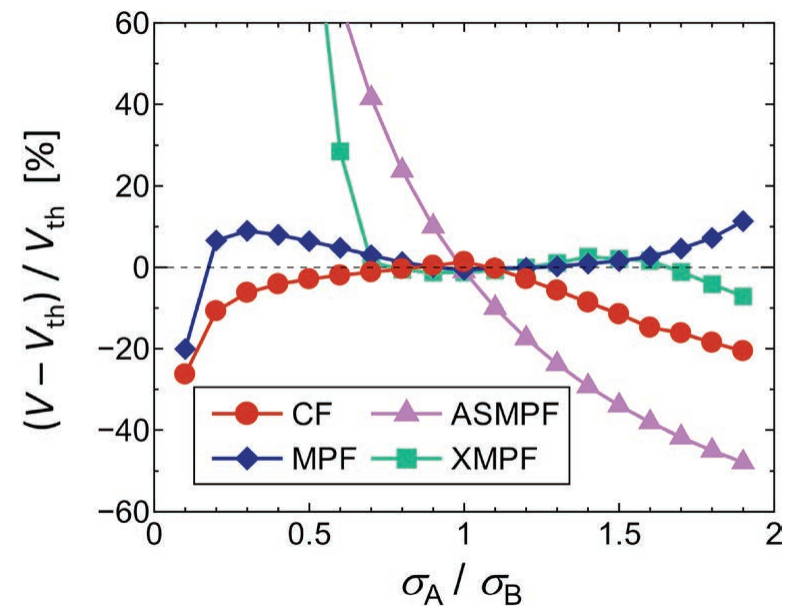

Fig. 3. Variations in relative error $\left(V-V_{\mathrm{th}}\right) / V_{\mathrm{th}}$ depending on boundary energy ratio $\sigma_{\mathrm{A}} / \sigma_{\mathrm{B}}$, as calculated from different phase-field models. (Online version in color.)
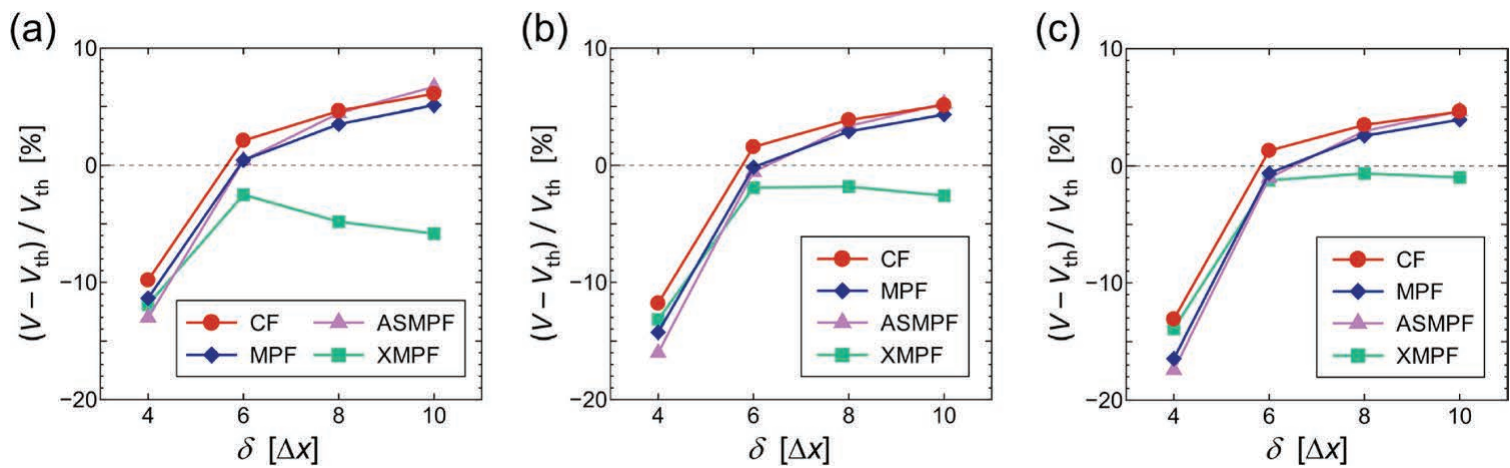

Fig. 2. Variations in relative error $\left(V-V_{\mathrm{th}}\right) / V_{\mathrm{th}}$ depending on grain boundary width $\delta$, as calculated from different phase-field models for grid resolutions of (a) $H / \Delta x=64$, (b) $H / \Delta x=96$, and (c) $H / \Delta x=128$. (Online version in color.) 
At $\sigma_{\mathrm{A}} / \sigma_{\mathrm{B}}=0.1$, the error jumps to the negative side, reaching $20 \%$. Therefore, if an error of $\sim 10 \%$ is acceptable, the MPF model is considered suitable for modeling grain growth under energy anisotropy within the ratio of the minimum-to-maximum boundary energy of 0.2 . Note that the accuracy of the MPF model obtained here is visibly better than that in our previous study; ${ }^{68)}$ this is because, as described in Section 2.2, the iterative algorithm of Takaki et $a .^{70)}$ was employed in the present MPF simulations, which significantly improves the computational accuracy. In Fig. 3, the CF model shows the similar degree of accuracy (magnitude of error) as that of the MPF model for $\sigma_{\mathrm{A}} / \sigma_{\mathrm{B}}<1$. However, for $\sigma_{\mathrm{A}} / \sigma_{\mathrm{B}}>1$, the decrease in the accuracy is relatively large, and the error is maximized at $\sim 20 \%$. In actual grain growth processes, triple junctions comprising one high-energy grain boundary and two low-energy boundaries, like the case of $\sigma_{\mathrm{A}} / \sigma_{\mathrm{B}}>1$ in Fig. 3 , are rarely formed. ${ }^{77-79)}$ Thus, in many cases, it is expected that the CF model can address anisotropic boundary energy with the similar degree of accuracy as that offered by the MPF model. In the ASMPF model, the increase in error is significant for both the ranges of $\sigma_{\mathrm{A}} / \sigma_{\mathrm{B}}>1$ and $\sigma_{\mathrm{A}} /$ $\sigma_{\mathrm{B}}<1$. Because the formulation of this model is simplified by ignoring the interactions of three or more grains, it is considered that the energy balance of grain boundaries at the triple junctions cannot be reproduced correctly. As an example, Fig. 4 shows the steady-state configurations of grain boundaries simulated by each phase-field model for the conditions $\sigma_{\mathrm{A}} / \sigma_{\mathrm{B}}=0.5$ and $\sigma_{\mathrm{A}} / \sigma_{\mathrm{B}}=1.5$. Unlike the cases with other models, the grain boundary angles in the ASMPF model are almost unchanged for the different energy ratios. Therefore, the ASMPF model is considered to be unsuitable for problems where grain boundary energy anisotropy is important. The XMPF model maintains less than $10 \%$ error in the range of $\sigma_{\mathrm{A}} / \sigma_{\mathrm{B}} \geq 0.7$. On the other hand, the error suddenly jumps to exceed $60 \%$ at $\sigma_{\mathrm{A}} / \sigma_{\mathrm{B}}=$ 0.5 . This sudden jump is attributed to the "ghost phase" problem, ${ }^{60,63)}$ via which unnecessary phases leak from the triple junction into the grain boundaries, thus inducing behavioral instabilities in the boundaries. As an example, Fig. 5 depicts the distributions of the values of phase field

(a)

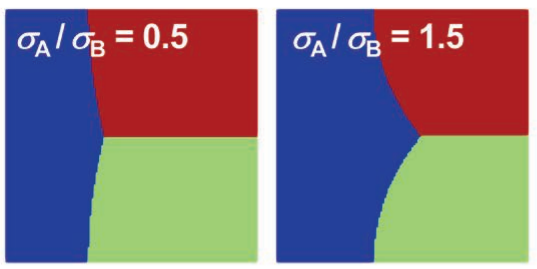

(b)
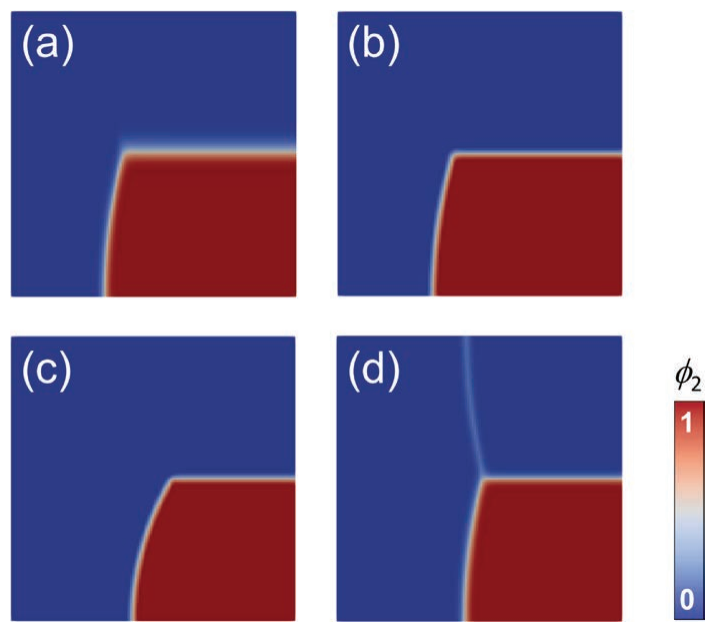

Fig. 5. Distributions of the values of phase field $\phi_{2}$ for the condition of $\sigma_{\mathrm{A}} / \sigma_{\mathrm{B}}=0.5$ : (a) CF model, (b) MPF model, (c) ASMPF model, and (d) XMPF model. In panel (d), the leakage of $\phi_{2}$ into the $\phi_{1}-\phi_{3}$ boundary (i.e., ghost phase) is observed. (Online version in color.)

$\phi_{2}$ for $\sigma_{\mathrm{A}} / \sigma_{\mathrm{B}}=0.5$, as obtained from the simulations using each phase-field model. As shown, for the XMPF model, the leakage of $\phi_{2}$ into the $\phi_{1}-\phi_{3}$ boundary is clearly observed.

\subsection{Introduction of Higher-order Term}

Tóth et al. ${ }^{63)}$ reported that the ghost phase problem of the XMPF model can be resolved by introducing a higher-order (triplet) term, $f_{\text {ho }}$, into the free energy functional:

$$
\begin{aligned}
F & =\int_{V}\left[f_{\text {barr }}(\phi)+f_{\text {grad }}(\phi, \nabla \phi)+f_{\text {ho }}(\phi)\right] \mathrm{d} V, \\
f_{\text {ho }} & =\sum_{i=1}^{N} \sum_{j>i}^{N} \sum_{k>j}^{N} C_{i j k} \phi_{i} \phi_{j} \phi_{k},
\end{aligned}
$$

where $C_{i j k}$ is a coefficient. The higher-order term energetically penalizes ghost phases, and is often used to improve the accuracy of other phase-field models. ${ }^{63-68)}$ Herein, by using the higher-order term, we investigate the possibility of improving the accuracies of the $\mathrm{CF}, \mathrm{MPF}$, and XMPF models. Note that the ASMPF model is not considered here because, in principle, this model cannot introduce the

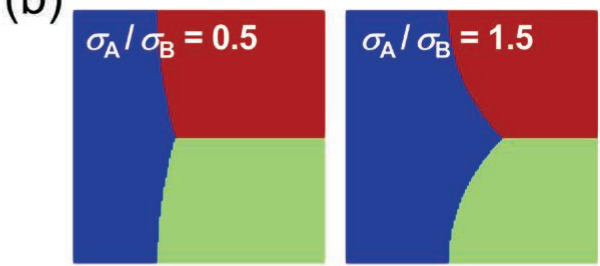

(d)
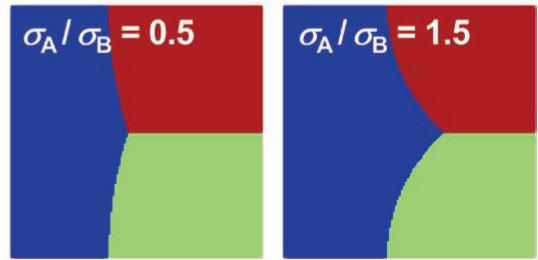

(c)

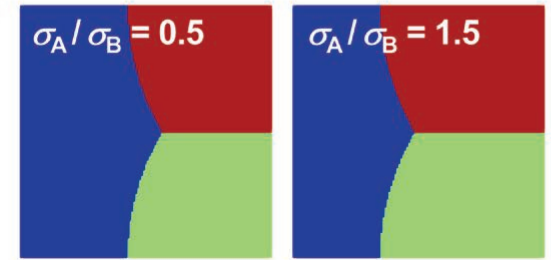

Fig. 4. Steady-state grain boundary configurations for the conditions of $\sigma_{\mathrm{A}} / \sigma_{\mathrm{B}}=0.5$ and $\sigma_{\mathrm{A}} / \sigma_{\mathrm{B}}=1.5$ : (a) CF model, (b) MPF model, (c) ASMPF model, and (d) XMPF model. In all panels, grains are distinguished by different colors. (Online version in color.) 
higher-order term because of the antisymmetric approximation.

The accuracy of the phase-field models with the higher-order term is largely dependent on the value of its coefficient, ${ }^{68)} C_{i j k}$. However, a way of setting $C_{i j k}$ to the appropriate value is not yet established. Therefore, to examine the optimum values of $C_{i j k}$, we first performed simulations using the system of Fig. 1 and each phase-field model for various energy ratios $\sigma_{\mathrm{A}} / \sigma_{\mathrm{B}}$, with $M_{\mathrm{A}}=M_{\mathrm{B}}$. For each $\sigma_{\mathrm{A}} /$ $\sigma_{\mathrm{B}}$ value, $C_{i j k}$ was varied by 0.01 , through which the variations in the relative error $\left(V-V_{\text {th }}\right) / V_{\text {th }}$ were determined. As an example, Fig. 6(a) shows the results of the XMPF model for $\sigma_{\mathrm{A}} / \sigma_{\mathrm{B}}=1$ and 0.5 . As shown, in both cases the relative error $\left(V-V_{\text {th }}\right) / V_{\text {th }}$ decreases almost monotonically with increases in $C_{i j k}$. For $\sigma_{\mathrm{A}} / \sigma_{\mathrm{B}}=0.5$, the magnitude of the relative error, $\left|\left(V-V_{\mathrm{th}}\right) / V_{\mathrm{th}}\right|$, approaches zero at $C_{i j k}=0.48$. For $\sigma_{\mathrm{A}} / \sigma_{\mathrm{B}}=1,\left|\left(V-V_{\mathrm{th}}\right) / V_{\mathrm{th}}\right|$ does not become zero, but is minimized at $C_{i j k}=0$. These values of $C_{i j k}$, which minimize $\left|\left(V-V_{\text {th }}\right) / V_{\text {th }}\right|$, are defined as the optimum values. The optimum values obtained for each model are summarized in Fig. $6(\mathrm{~b})$ as functions of $\sigma_{\mathrm{A}} / \sigma_{\mathrm{B}}$.

Next, we compared the accuracy of the models with and without the higher-order term via accuracy evaluation simulations, in the same manner as described in Section 3.2. Here, the coefficients of the higher-order terms use the optimal values shown in Fig. 6(b). The results are given in Fig. 7. (a)

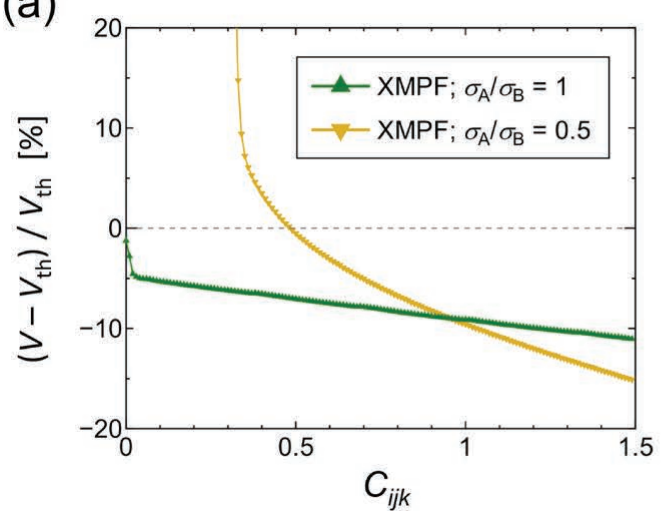

(b)

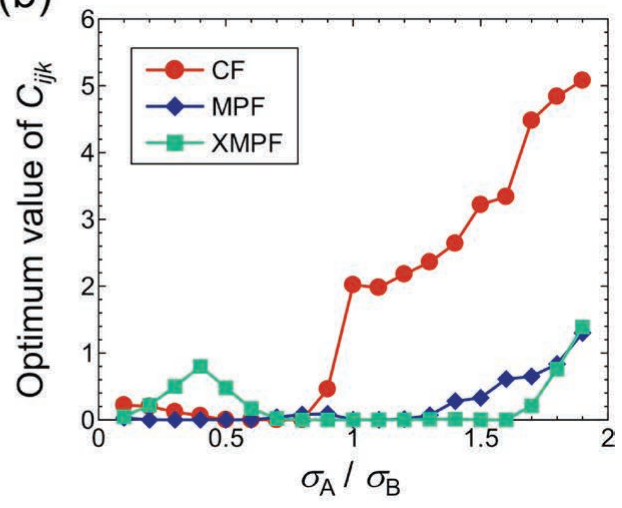

Fig. 6. Determining the optimum values of $C_{i j k}$. (a) Variations in $\left(V-V_{\mathrm{th}}\right) / V_{\text {th }}$ depending on $C_{i j k}$, as calculated from the XMPF model for $\sigma_{\mathrm{A}} / \sigma_{\mathrm{B}}=1$ and $\sigma_{\mathrm{A}} / \sigma_{\mathrm{B}}=0.5$. (b) Variations in the optimum value of $C_{i j k}$ for minimizing $\mid(V-$ $\left.V_{\mathrm{th}}\right) / V_{\mathrm{th}} \mid$ for different $\sigma_{\mathrm{A}} / \sigma_{\mathrm{B}}$ values. (Online version in color.)

(a)

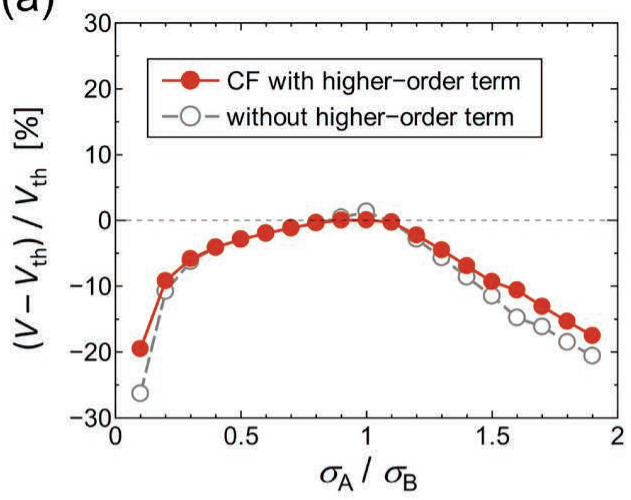

(b)

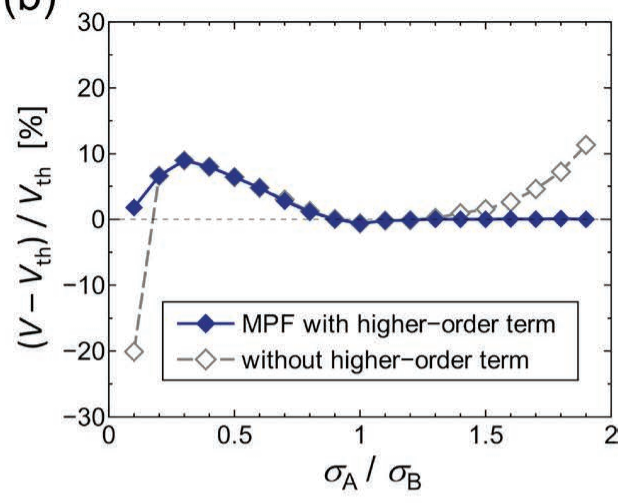

(c)

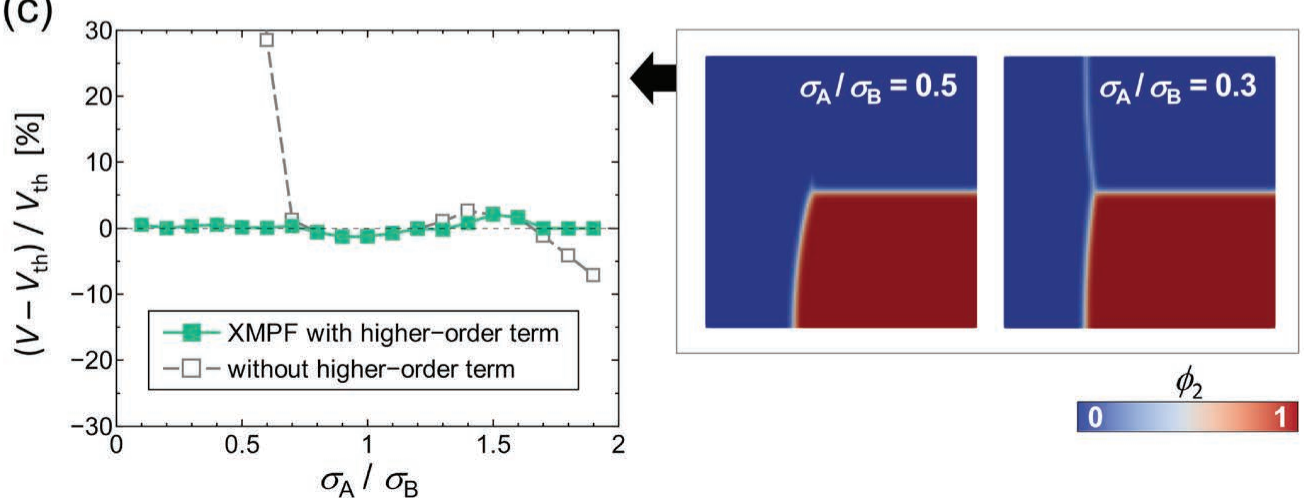

Fig. 7. Variations in $\left(V-V_{\text {th }}\right) / V_{\text {th }}$ with and without the higher-order term for different $\sigma_{\mathrm{A}} / \sigma_{\mathrm{B}}$ values: (a) CF model, (b) MPF model, and (c) XMPF model. The right panel of (c) depicts the distributions of the values of phase field $\phi_{2}$ for $\sigma_{\mathrm{A}} / \sigma_{\mathrm{B}}=0.5$ and $\sigma_{\mathrm{A}} / \sigma_{\mathrm{B}}=0.3$, as calculated using the XMPF model with the higher-order term. the coefficients of the higher-order terms use the optimal values shown in Fig. 6 (b). (Online version in color.) 


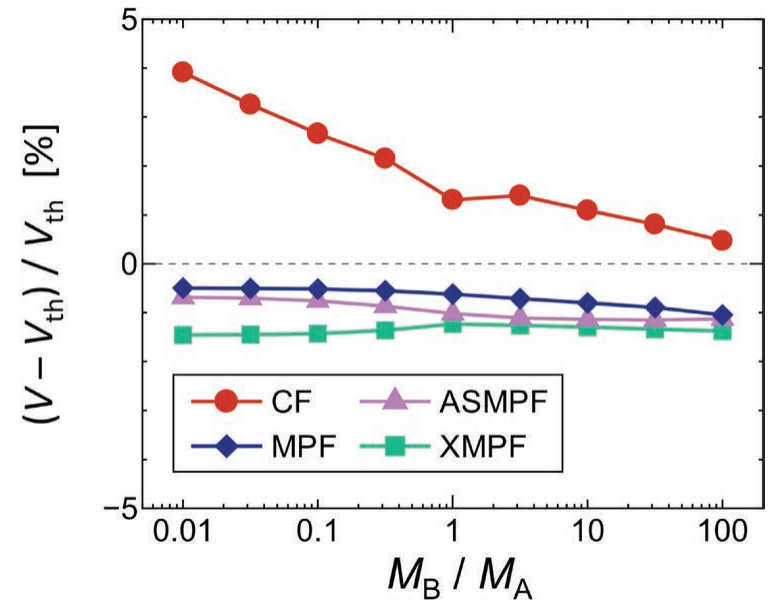

Fig. 8. Variations in relative error $\left(V-V_{\text {th }} / V_{\text {th }}\right.$ depending on boundary mobility ratio $M_{\mathrm{B}} / M_{\mathrm{A}}$, as calculated from different phase-field models. (Online version in color.)

As can be seen from Fig. 7(a), the accuracy improvement for the CF model is small. In Fig. 7(b), The MPF model can reduce the error for $\sigma_{\mathrm{A}} / \sigma_{\mathrm{B}}>1.3$ and $\sigma_{\mathrm{A}} / \sigma_{\mathrm{B}}=0.1$ to almost zero by including the higher-order term, but there is no improvement for other ranges of $\sigma_{\mathrm{A}} / \sigma_{\mathrm{B}}$. In Fig. 7(c) for the XMPF model, the higher-order term suppresses the error to almost zero for most of the grain boundary energy ratios, exhibiting a drastic improvement in accuracy. However, even though the coefficient $C_{i j k}$ is set to the optimum value (Fig. 6(b)), the ghost phase problem still occurs in the range of $\sigma_{\mathrm{A}} / \sigma_{\mathrm{B}} \leq 0.3$, as depicted in the right panel of Fig. $7(\mathrm{c})$. By increasing $C_{i j k}$ from the optimum value for $\sigma_{\mathrm{A}} / \sigma_{\mathrm{B}}=$ 0.3 , the disappearance of the ghost phase is observed at $C_{i j k}$ approximately twice the optimum value; nevertheless, the error $\left(V-V_{\text {th }}\right) / V_{\text {th }}$ reaches $40 \%$, resulting in the decrease of the computational accuracy for the grain boundary velocity. To fully achieve both accuracy and ghost phase suppression, additional improvement of the model might be required.

\subsection{Effect of Anisotropic Grain Boundary Mobility}

Finally, we evaluated the accuracy of the phase-field models while considering anisotropic grain boundary mobilities. Here, grain growth simulations using the system of Fig. 1 and different phase-field models were performed for various mobility ratios $M_{\mathrm{B}} / M_{\mathrm{A}}$, with $\sigma_{\mathrm{A}} / \sigma_{\mathrm{B}}$ fixed to 1 . The results are shown in Fig. 8, where the relative errors $\left(V-V_{\text {th }}\right) / V_{\text {th }}$ are plotted as functions of $M_{\mathrm{B}} / M_{\mathrm{A}}$. As shown in the figure, the MPF, ASMPF, and XMPF models maintain almost constant errors of less than $2 \%$ for the very wide-ranging mobility ratio range of $0.01 \leq M_{\mathrm{B}} / M_{\mathrm{A}} \leq 100$, exhibiting high degrees of accuracy. Compared to these models, the increase in the error of the CF model is relatively large, especially for $M_{\mathrm{B}} /$ $M_{\mathrm{A}}<1$; however, the maximum error remains within $4 \%$. These results enable us to conclude that, if we accept an error of several percent, all of the phase-field models allow for the introduction of arbitrarily strong anisotropy in grain boundary mobility.

\section{Conclusions}

This study was conducted to elucidate the applicability of four different phase-field models in simulating grain growth under conditions of strongly anisotropic grain boundary energy and mobility. To this end, the accuracies of the phase-field models were systematically evaluated by performing a series of anisotropic grain growth simulations. In addition, based on the higher-order modification, accuracy improvement of the models was attempted. The main findings are summarized as follows:

(1) All of the phase-field models can be used for grain growth simulations with almost arbitrarily strong mobility anisotropy.

(2) If an error of around $10 \%$ is accepted, the CF and MPF models without the higher-order term can adequately simulate energy anisotropies within the ratio of the minimum-to-maximum boundary energies $\sigma_{\min } / \sigma_{\max }=0.2$. Although the usage of the higher-order term improves the accuracy of the MPF model for simulations with stronger energy anisotropy, errors near $10 \%$ are not fully resolved.

(3) By using the higher-order term, the XMPF model achieves high accuracy with almost no error for any grain boundary energy ratio. However, to achieve both accuracy and ghost phase suppression for the range of $\sigma_{\min } / \sigma_{\max } \leq 0.3$, further improvement of the model is required.

(4) The ASMPF model is not suitable for simulations with anisotropic boundary energy. However, because of its simple formulation and resultant computational efficiency, this model is a promising choice for conditions of only anisotropic mobility.

\section{REFERENCES}

1) F. J. Humphreys and M. Hatherly: Recrystallisation and Related Annealing Phenomena, 2nd ed., Elsevier, Oxford, (2004), 333.

2) H. V. Atkinson: Acta Metall., 36 (1988), 469.

3) C. V. Thompson: Solid State Phys., 55 (2000), 269

4) M. A. Miodownik: J. Light Met., 2 (2002), 125.

5) M. P. Anderson, D. J. Srolovitz, G. S. Grest and P. S. Sahni: Acta Metall., 32 (1984), 783.

6) D. J. Srolovitz, M. P. Anderson, P. S. Sahni and G. S. Grest: Acta Metall., 32 (1984), 793.

7) Y. Suwa and Y. Saito: Mater. Trans., 46 (2005), 1214

8) J. K. Mason: Acta Mater., 94 (2015), 162.

9) J. Geiger, A. Roósz and P. Barkóczy: Acta Mater., 49 (2001), 623.

10) Y. He, H. Ding, L. Liu and K. Shin: Mater. Sci. Eng. A, 429 (2006), 236.

11) H. L. Ding, Y. Z. He, L. F. Liu and W. J. Ding: J. Cryst. Growth, 293 (2006), 489

12) H. J. Frost, C. V. Thompson, C. L. Howe and J. Whang: Scr. Metall, 22 (1988), 65

13) K. Kawasaki and T. Okuzono: Phase Transit., 28 (1990), 177.

14) K. Fuchizaki, T. Kusaba and K. Kawasaki: Philos. Mag. B, 71 (1995), 333.

15) D. Weygand, Y. Bréchet and J. Lépinoux: Philos. Mag. B, 78 (1998), 329.

16) D. Weygand, Y. Bréchet, J. Lépinoux and W. Gust: Philos. Mag. B, 79 (1999), 703.

17) J. K. Mason, E. A. Lazar, R. D. MacPherson and D. J. Srolovitz: Phys. Rev. E, 92 (2015), 063308.

18) K. A. Brakke: Exp. Math., 1 (1992), 141

19) F. Wakai, N. Enomoto and H. Ogawa: Acta Mater., 48 (2000), 1297.

20) S. Jurine, S. Cox and F. Graner: Colloids Surf. A, 263 (2005), 18.

21) C. E. Krill III and L.-Q. Chen: Acta Mater., 50 (2002), 3059.

22) S. G. Kim, D. I. Kim, W. T. Kim and Y. B. Park: Phys. Rev. E, 74 (2006), 061605.

23) M. Ohno, S. Tsuchiya and K. Matsuura: Acta Mater., 59 (2011), 5700 .

24) R. Darvishi Kamachali and I. Steinbach: Acta Mater., 60 (2012), 2719.

25) R. Perumal, P. G. Kubendran Amos, M. Selzer and B. Nestler: Comput. Mater. Sci., 147 (2018), 227.

26) V. Yadav and N. Moelans: Scr. Mater., 142 (2018), 148.

27) Y. Shibuta, M. Ohno and T. Takaki: JOM, 67 (2015), 1793.

28) T. Takaki, S. Sakane, M. Ohno, Y. Shibuta, T. Shimokawabe and T. Aoki: Acta Mater., 118 (2016), 230

29) E. Miyoshi, T. Takaki, M. Ohno, Y. Shibuta, S. Sakane, T. 
Shimokawabe and T. Aoki: npj Comput. Mater., 3 (2017), 25.

30) Y. Shibuta, M. Ohno and T. Takaki: Adv. Theory Simul., 1 (2018), 1800065

31) E. Miyoshi, T. Takaki, M. Ohno, Y. Shibuta, S. Sakane, T. Shimokawabe and T. Aoki: J. Mater. Sci., 53 (2018), 15165.

32) T. Takaki, S. Sakane, M. Ohno, Y. Shibuta, T. Aoki and C.-A. Gandin: Materialia, 1 (2018), 104.

33) T. Takaki, S. Sakane, M. Ohno, Y. Shibuta and T. Aoki: Acta Mater., 164 (2018), 237.

34) G. Kim, T. Takaki, Y. Shibuta, S. Sakane, K. Matsuura and M. Ohno: Comput. Mater. Sci., 162 (2019), 76.

35) G. Gottstein and L. S. Shvindlerman: Grain Boundary Migration in Metals: Thermodynamics, Kinetics, Applications, CRC Press, Boca Raton, (1999), 111.

36) B. L. Adams, D. Kinderlehrer, W. W. Mullins, A. D. Rollett and S. Ta'asan: Scr. Mater., 38 (1998), 531.

37) C. C. Yang, A. D. Rollett and W. W. Mullins: Scr. Mater., 44 (2001), 2735.

38) A. D. Rollett, D. J. Srolovitz and M. P. Anderson: Acta Metall., 37 (1989), 1227

39) F. J. Humphreys: Acta Mater., 45 (1997), 4231

40) E. A. Holm, M. A. Miodownik and A. D. Rollett: Acta Mater., 51 (2003), 2701 .

41) O. M. Ivasishin, S. V. Shevchenko and S. L. Semiatin: Scr. Mater., 50 (2004), 1241.

42) A. D. Rollett: JOM, 56 (2004), 63.

43) S. M. Na and A. B. Flatau: Smart Mater. Struct., 22 (2013), 125026.

44) J. Gruber, D. C. George, A. P. Kuprat, G. S. Rohrer and A. D. Rollett: Scr. Mater., 53 (2005), 351.

45) G. S. Rohrer: Annu. Rev. Mater. Res., 35 (2005), 99.

46) J. Gruber, H. M. Miller, T. D. Hoffmann, G. S. Rohrer and A. D. Rollett: Acta Mater., 57 (2009), 6102.

47) E. A. Holm, G. N. Hassold and M. A. Miodownik: Acta Mater., 49 (2001), 2981.

48) S. Okita, E. Miyoshi, S. Sakane, T. Takaki, M. Ohno and Y. Shibuta: Acta Mater., 153 (2018), 108.

49) L.-Q. Chen and W. Yang: Phys. Rev. B, 50 (1994), 15752.

50) D. Fan and L.-Q. Chen: Acta Mater., 45 (1997), 3297.

51) D. Fan and L.-Q. Chen: Acta Mater., 45 (1997), 611.

52) A. Kazaryan, Y. Wang, S. A. Dregia and B. R. Patton: Phys. Rev. B, 63 (2001), 184102.

53) N. Moelans, B. Blanpain and P. Wollants: Phys. Rev. B, 78 (2008), 024113.

54) N. Moelans, B. Blanpain and P. Wollants: Phys. Rev. Lett., 101
(2008), 025502.

55) I. Steinbach, F. Pezzolla, B. Nestler, M. Seeßelberg, R. Prieler, G. J. Schmitz and J. L. L. Rezende: Physica D, 94 (1996), 135.

56) H. Garcke, B. Nestler and B. Stoth: SIAM J. Appl. Math., 60 (1999), 295.

57) I. Steinbach and F. Pezzolla: Physica D, 134 (1999), 385.

58) J. Eiken, B. Böttger and I. Steinbach: Phys. Rev. E, 73 (2006), 066122.

59) G. I. Tóth, T. Pusztai and L. Gránásy: Phys. Rev. B, 92 (2015), 184105 .

60) N. Moelans, F. Wendler and B. Nestler: Comput. Mater. Sci., 46 (2009), 479

61) W. Guo and I. Steinbach: Int. J. Mater. Res., 101 (2010), 480.

62) Y. Nishida and S. Itoh: Acta Mater., 60 (2012), 4077.

$63)$ G. I. Tóth, M. Zarifi and B. Kvamme: Phys. Rev. E, 93 (2016), 013126.

64) T. Takaki, T. Hirouchi and Y. Tomita: J. Cryst. Growth, 310 (2008), 2248.

65) P. C. Bollada, P. K. Jimack and A. M. Mullis: Physica D, 241 (2012), 816.

66) T. Hirouchi, T. Tsuru and Y. Shibutani: Comput. Mater. Sci., 53 (2012), 474

67) K. Ankit, B. Nestler, M. Selzer and M. Reichardt: Contrib. Mineral. Petrol., 166 (2013), 1709.

68) E. Miyoshi and T. Takaki: Comput. Mater. Sci., 112 (2016), 44.

69) M. Tang, B. W. Reed and M. Kumar: J. Appl. Phys., 112 (2012), 043505 .

70) T. Takaki, T. Hirouchi, Y. Hisakuni, A. Yamanaka and Y. Tomita: Mater. Trans., 49 (2008), 2559.

71) J. Tiadan, B. Nestler, H. J. Diepers and I. Steinbach: Physica D, 115 (1998), 73.

72) E. Brosh and R. Z. Shneck: J. Am. Ceram. Soc., 87 (2004), 640.

$73)$ N.-M. Hwang, Y.-C. Joo, C. H. Han, D.-Y. Kim, H. Park and J.-K. Kim: J. Appl. Phys., 95 (2004), 5515.

74) M. Ohno, T. Takaki and Y. Shibuta: J. Comput. Phys., 335 (2017), 621 .

75) J. Gruber, N. Ma, Y. Wang, A. D. Rollett and G. S. Rohrer: Model. Simul. Mater. Sci. Eng., 14 (2006), 1189.

76) S. Vedantam and B. S. V. Patnaik: Phys. Rev. E, 73 (2006), 016703.

77) O. V. Mishin and G. Gottstein: Mater. Sci. Eng. A, 249 (2002), 71.

78) M. Y. Huh, L. A. Barrales-Mora and O. Engler: Int. J. Mater. Res., 103 (2012), 1423.

79) Y. B. Zhang, T. Yu and O. V. Mishin: Mater. Lett., 186 (2017), 102. 